\title{
Clinical Features and Outcomes of a Racially Diverse Population with Fibrillary Glomerulonephritis
}

\author{
Fernanda Payan Schober ${ }^{a}$ Meghan A. Jobson ${ }^{a}$ b Caroline J. Poulton ${ }^{a}$ \\ Harsharan K. Singh ${ }^{c}$ Volker Nickeleit ${ }^{c}$ Ronald J. Falk ${ }^{a, b} \quad$ J. Charles Jennette ${ }^{b, c}$ \\ Patrick H. Nachman ${ }^{a, b}$ William F. Pendergraft III ${ }^{a, b}$ \\ a University of North Carolina (UNC) Kidney Center, Division of Nephrology and Hypertension, Department of \\ Medicine, ${ }^{b}$ UNC School of Medicine, and ' UNC Division of Nephropathology, Department of Pathology and \\ Laboratory Medicine, Chapel Hill, NC, USA
}

\section{Keywords}

Fibrillary glomerulonephritis · Hepatitis C · Black or African American

\begin{abstract}
Background: Fibrillary glomerulonephritis is characterized by randomly arranged fibrils, approximately $20 \mathrm{~nm}$ in diameter by electron microscopy. Patients present with proteinuria, hematuria and kidney insufficiency, and about half of the reported patients progress to end-stage kidney disease within 4 years. The dependence of patient characteristics and outcomes on race has not been explored. In this study, we describe a cohort of patients with fibrillary glomerulonephritis and compare their clinical characteristics and outcomes with those of patients previously described. Methods: The University of North Carolina (UNC) Nephropathology Database was used to retrospectively identify patients diagnosed with fibrillary glomerulonephritis between 1985 and 2015. Of these patients, those treated at UNC were selected. Their demographic and clinical characteristics - including signs and symptoms, comorbidities, laboratory values, treatments and outcomes - were compared with those of patients described earlier. Results: Among the 287 patients identified, 42 were treated at the UNC Kidney Center.
\end{abstract}

When compared to earlier cohorts, a higher frequency of black race, hepatitis $C$ virus (HCV) infection and use of hemodialysis were noted in both black and HCV-positive patients. Autoimmune diseases, infections and malignancies were frequently observed, present in over half of all cases. Conclusion: According to this study, fibrillary glomerulonephritis represents a secondary glomerular disease process (associated with autoimmune disease, infection or malignancy) in many cases and hence screening is essential. As the screening for comorbidities increased over time, more underlying causes were identified. We noted a high frequency of HCV among black patients, suggesting a possible causative association. Treatment of underlying disease is essential for patients for the best outcome. $\odot 2017$ S. Karger AG, Basel

\section{Introduction}

Fibrillary glomerulonephritis is a rare glomerular disease found in less than $1 \%$ of native kidney biopsies $[1,2]$. Initially described in 1977, and later refined, fibrillary glo-

F.P.S. and M.A.J. contributed equally to this work.

\section{KARGER}

(C) 2017 S. Karger AG, Basel

E-Mail karger@karger.com

www.karger.com/ajn
Dr. William F. Pendergraft III, MD, PhD 
merulonephritis is characterized by the presence of randomly arranged fibrils measuring approximately $20 \mathrm{~nm}$ in diameter by electron microscopy [3-5]. These fibrils are Congo red negative and infrequently identified in the vascular walls or interstitium, distinguishing them from amyloid deposits. Light microscopy reveals a spectrum of injury patterns, including diffuse mesangial or endocapillary proliferation, with crescents identified in approximately $25 \%$ of cases [6]. Immunofluorescence microscopy typically demonstrates IgG dominance (predominantly IgG4) with positive C3 staining [7].

Treatment is challenging and approximately half of all patients progress to end-stage kidney disease (ESKD) within 4 years of diagnosis $[1,2,4,6,8]$. Although fibrillary glomerulonephritis has been associated with autoimmune disorders, infections and malignancy [9-15], it is traditionally considered a primary, or "idiopathic," glomerular disease - in contrast to immunotactoid glomerulopathy, which is characterized by the presence of larger microtubules arranged in parallel and is strongly associated with lymphoproliferative disorders [1, 16-19]. Relationships between clinical characteristics, region of residence and race have rarely been explored in fibrillary glomerulonephritis.

In this report, we describe the clinical characteristics (including clinical presentation, treatments, outcomes and associations with non-renal diseases) of a cohort of racially diverse patients with fibrillary glomerulonephritis residing in the southeastern United States, and compare our findings with those previously reported.

\section{Subjects and Methods}

\section{Settings and Participants}

Patients diagnosed with fibrillary glomerulonephritis by native kidney biopsy between 1985 and 2015 were identified from the University of North Carolina (UNC) Nephropathology Database and the Glomerular Disease Collaborative Network (GDCN), a partnership involving the UNC Kidney Center, the UNC Division of Nephropathology and community practices in the southeastern United States. This study (UNC IRB \#15-1252) was approved by the UNC Institutional Review Board (IRB).

\section{Design Overview, Definitions and Data Analysis}

The diagnosis of fibrillary glomerulonephritis was based on the presence of randomly arranged non-branching fibrils $10-30 \mathrm{~nm}$ in diameter, deposited in the mesangium and capillary walls, by electron microscopy. The diagnosis was supported by positive immunoglobulin $\mathrm{G}$ staining and/or absence of Congo red staining by immunofluorescence microscopy. Biopsies must not have histopathological features, indicative of cryoglobulinemic glomerulonephritis or immunotactoid glomerulopathy. Patients were diagnosed with monoclonal gammopathy of undetermined significance (MGUS) if they had a monoclonal protein present on serum

Clinical Features and Outcomes of Fibrillary GN protein electrophoresis or immunofixation, but did not meet diagnostic criteria for multiple myeloma. They were classified as having monoclonal gammopathy of renal significance (MGRS) if they did not meet the criteria for multiple myeloma, but a hematologist considered their monoclonal gammopathy to be a contributing factor to kidney dysfunction.

We retrospectively extracted demographic and clinical data from patient medical records, including presenting signs and symptoms, comorbidities, laboratory values, treatment and outcomes at the time of kidney biopsy. More recent kidney function status was classified as non-progressive (serum creatinine remained stable, improved or increased $<25 \%$ of the original value after treatment), progressive (serum creatinine increased $>25 \%$ of the original value during biopsy) or $\operatorname{ESKD}[3,20,21]$. Primary outcomes were reported based on the last available clinic visit or report (as death, dialysis, transplant or doubling of serum creatinine). Data were compared with published cohorts of patients with fibrillary glomerulonephritis found on PubMed using the $\mathrm{MeSH}$ terms "glomerulonephritis" and "fibrillary" combined with Boolean operator "AND” to maximize sensitivity.

\section{Statistical Analysis}

Statistical analysis was performed using Prism 5.0 (GraphPad). Continuous variables were reported as means with SDs and compared with the Mann-Whitney test. Categorical variables, reported as frequencies and percentages, were compared with the Fisher's exact test or chi-square as appropriate. Kaplan-Meier survival analysis was used to compare time to kidney impairment. A twosided significance level of $p<0.05$ was assumed.

\section{Results}

\section{Demographic and Clinical Characteristics}

Between 1985 and 2015, 287 cases with a histopathological diagnosis of fibrillary glomerulonephritis were evaluated by UNC nephropathologists, and the UNC Kidney Center prescribed treatment for 42 patients (Table 1). Demographic and clinical characteristics of patients evaluated at the UNC Kidney Center were comparable to those of the entire biopsied cohort.

Demographic, laboratory and comorbidity characteristics at the time of biopsy are summarized in Table 2 for patients who were prescribed treatment at the UNC Kidney Center. Most patients had hematuria (95\%), proteinuria (97\%) and kidney impairment (mean creatinine 3.2 $\mathrm{mg} / \mathrm{dL}$ ) at presentation. Serum creatinine was found to be higher in black patients compared to white patients ( $4.7 \mathrm{vs.}$ $2.7 \mathrm{mg} / \mathrm{dL}, p=0.01$ ), and black patients were more likely to have transitioned to dialysis ( 82 vs. $30 \%, p=0.004$ ). Hepatitis $\mathrm{C}$ virus (HCV) infection was more common in black patients compared to white patients ( 7 vs. 0 patients, $p<$ $0.001)$. There were no statistical differences based on sex in our population (online suppl. Table 1; for all online suppl. material, see www.karger.com/doi/10.1159/000455390). 
Table 1. The UNC Kidney Center cohort of patients with fibrillary glomerulonephritis is a representative sample of the entire cohort of patients within the UNC Nephropathology database

\begin{tabular}{llc}
\hline & $\begin{array}{l}\text { UNC nephropathology } \\
2015(n=245)\end{array}$ & $\begin{array}{l}\text { UNC Kidney Center } \\
2015(n=42)\end{array}$ \\
\hline Age, years & $57 \pm 12$ & $54 \pm 10$ \\
Race, \% & & \\
$\quad$ White & 68 & 71 \\
$\quad 16$ & 26 \\
$\quad$ Black & 16 & 2 \\
Femaler/unknown $\%$ & 62 & 60 \\
Serum creatinine, $\mathrm{mg} / \mathrm{dL}$ & $3.3 \pm 3(155)$ & $3.0 \pm 3(36)$ \\
eGFR, mL/min/1.73 m ${ }^{2}$ & $34 \pm 27(155)$ & $35 \pm 20(35)$ \\
Albumin, g/dL & $3.1 \pm 1(77)$ & $3.1 \pm 1(23)$ \\
Proteinuria, g/24h & $4.8 \pm 4(113)$ & $5.7 \pm 5(33)$ \\
\hline
\end{tabular}

Further evaluation of the HCV-positive patient population determined that these patients were younger than the HCV-negative patients ( 50 vs. 58 years, $p=0.02$ ) and were more likely to be black ( 100 vs. $16 \%, p<0.001$; Table 2). Although the status of hepatitis was not tested for all patients, HCV infection was more common at the UNC Kidney Center compared to previously published data (26\%; Table 3$)$. Serum cryoglobulins were measured and undetectable in 4 of the 7 patients who were $\mathrm{HCV}$ positive. None of the 7 patients had diagnostic features of cryoglobulinemic glomerulonephritis on biopsy. Serum albumin was similar in $\mathrm{HCV}$-positive and $\mathrm{HCV}$-negative patients ( 2.9 vs. $3.2 \mathrm{~g} / \mathrm{dL}, p=0.66)$ and $\mathrm{C} 3$ was not substantially lowered in $\mathrm{HCV}$-positive patients, suggesting that the liver function was not severely altered. This was further supported by normal liver function tests and/or absence of cirrhosis by imaging in these patients. HCVpositive patients were more likely to be transitioned to dialysis than patients with negative $\mathrm{HCV}$ serologies (89 vs. $26 \%, p=0.02$ ).

A review of the literature revealed 6 earlier reports of cohorts containing 9 or more patients with fibrillary glomerulonephritis, along with one study that summarized published reports between 1977 and 1994 (online suppl. Table 2). A qualitative comparison of patients in these published cohorts with those in our cohort showed similar laboratory values at the time of biopsy including serum creatinine (online suppl. Table 2). The cohort in the study had a higher percentage of black patients ( 28 vs. 0-10\%) compared to other cohorts, reflecting local demographics. Comorbidities in our cohort were similar to those previously reported, with the exception of $\mathrm{HCV}$, which was more common in our cohort; however, many studies did not report comorbidities (Table 3). Fifty-eight percent of our patients had a concomitant autoimmune disorder, infection or malignancy.

Autoimmune disorders have been identified in other cohorts, affecting $3-30 \%$ of patients with fibrillary glomerulonephritis. Ten percent of patients in our cohort had an autoimmune disorder. Six patients (12\%) had a solid tumor malignancy (including basal cell and squamous cell carcinoma of the skin, renal carcinoma, breast carcinoma and non-squamous cell lung carcinoma). There were 4 patients with MGUS and 2 patients with MGRS treated with a multiple myeloma chemotherapy regimen. Of note, none of these patients had monoclonal immunoglobulin deposition on immunofluorescence microscopy.

\section{Histopathology}

The average number of glomeruli examined on light microscopy was 18 (range 4-67), with an average of $29 \%$ glomeruli globally sclerosed and $5 \%$ crescentic. The percentage of globally sclerotic glomeruli was increased in black patients ( $39 \%$ vs. non-black $25 \%, p=0.18$ ), HCVpositive patients ( $34 \%$ vs. HCV negative $25 \%, p=0.71)$, and patients who progressed to ESKD ( 41 vs. $22 \%, p=$ $0.02)$. Interstitial fibrosis and tubular atrophy (IFTA) were observed in $92 \%$ of kidney biopsies (characterized as $26 \%$ mild, $40 \%$ moderate, $26 \%$ severe). IFTA was more severe in patients with outcomes of ESKD ( $p=0.03$ vs. those without). Differences in IFTA between black and non-black patients $(p=0.06)$ and HCV-negative and HCV-positive patients $(p=0.28)$ were not significant. Similarly, arteriosclerosis increased in patients with outcomes of $\operatorname{ESKD}(p=0.01)$, while there were no significant differences based on race $(p=0.16)$ and HCV status $(p=$ 0.22 ). Immunofluorescence staining pattern was positive 
Table 2. Differences between patients with fibrillary glomerulonephritis based on race and hepatitis C virus status at UNC Kidney Center

\begin{tabular}{|c|c|c|c|c|c|c|c|}
\hline & $\begin{array}{l}\text { UNC Kidney } \\
\text { Center } \\
(n=42)\end{array}$ & $\begin{array}{l}\text { Black } \\
(n=11)\end{array}$ & $\begin{array}{l}\text { White } \\
(n=30)\end{array}$ & $p$ value & $\begin{array}{l}\operatorname{HCV}(-) \\
(n=19)\end{array}$ & $\begin{array}{l}\mathrm{HCV}(+) \\
(n=7)\end{array}$ & $p$ value $^{\dagger}$ \\
\hline \multicolumn{8}{|l|}{ Demographics } \\
\hline Age, years & $54 \pm 11$ & $49 \pm 8$ & $56 \pm 11$ & 0.06 & $58 \pm 12$ & $50 \pm 3$ & 0.02 \\
\hline Female, \% & 60 & 46 & 63 & 0.49 & 63 & 29 & 0.19 \\
\hline Black race, $\%$ & 26 & - & - & & 16 & 100 & $<0.001$ \\
\hline Serum creatinine, $\mathrm{mg} / \mathrm{dL}$ & $3.2 \pm 4(38)$ & $4.7 \pm 4$ & $2.7 \pm 4(26)$ & 0.01 & $3.4 \pm 4$ & $2.9 \pm 0.9$ & 0.31 \\
\hline $\mathrm{eGFR}, \mathrm{mL} / \mathrm{min} / 1.73^{2}$ & $35 \pm 20(38)$ & $25 \pm 17$ & $36 \pm 23(25)$ & 0.11 & $31 \pm 20$ & $29.5 \pm 15$ & 0.96 \\
\hline Albumin, $\mathrm{g} / \mathrm{dL}$ & $3.1 \pm 1(24)$ & $2.8 \pm 0.9$ & $3.5 \pm 0.8(12)$ & 0.08 & $3.2 \pm 0.9(13)$ & $2.9 \pm 1$ & 0.66 \\
\hline Proteinuria, g/24 h & $5.7 \pm 5(33)$ & $7.0 \pm 4.6(9)$ & $5.5 \pm 4.8(24)$ & 0.24 & $7.0 \pm 5(18)$ & $6.4 \pm 5(6)$ & 0.97 \\
\hline Proteinuria (any) & $95(36)$ & $100(11)$ & $96(24)$ & 1 & 100 & $100(6)$ & 1 \\
\hline Hematuria (any) & $97(37)$ & $100(11)$ & $92(25)$ & 1 & 100 & $100(6)$ & 1 \\
\hline $\mathrm{TG}, \mathrm{mg} / \mathrm{dL}$ & $186 \pm 106(9)$ & $164 \pm 111(5)$ & $428 \pm 425(4)$ & 0.29 & $457 \pm 397(4)$ & $155 \pm 127(4)$ & 0.11 \\
\hline Systolic BP, mm Hg & $141 \pm 19(34)$ & $144 \pm 23$ & $140 \pm 18(22)$ & 0.86 & $143 \pm 20(18)$ & $140 \pm 21$ & 0.63 \\
\hline Diastolic BP, mm Hg & $82 \pm 11(34)$ & $86 \pm 12$ & $80 \pm 10(22)$ & 0.13 & $79 \pm 12(18)$ & $84 \pm 8$ & 0.35 \\
\hline $\mathrm{WBC}, 10^{9} / \mathrm{L}$ & $7.4 \pm 3(21)$ & $7.5 \pm 2.8(9)$ & $6.8 \pm 2.3(11)$ & 0.82 & $6.9 \pm 3(12)$ & $7.4 \pm 2.5$ & 0.90 \\
\hline $\mathrm{HCT}, \%$ & $31 \pm 8(19)$ & $30 \pm 9(8)$ & $32 \pm 7(10)$ & 0.97 & $30 \pm 9(12)$ & $34 \pm 5.5(6)$ & 0.37 \\
\hline Hemoglobin, g/dL & $10.9 \pm 2(23)$ & $10.6 \pm 1.4(10)$ & $11.2 \pm 2.5(12)$ & 0.74 & $10.6 \pm 2.2(14)$ & $11 \pm 2.5$ & 0.39 \\
\hline Platelets, $10^{9} / \mathrm{L}$ & $256 \pm 95(22)$ & $251 \pm 105(10)$ & $262 \pm 96(11)$ & 0.81 & $240 \pm 112(12)$ & $275 \pm 86$ & 0.64 \\
\hline ANA, $\%(n)$ & $41(29)$ & $40(10)$ & $42(19)$ & 1 & 42 & $50(6)$ & 1 \\
\hline $\mathrm{C} 3, \mathrm{mg} / \mathrm{dL}$ & $113 \pm 29(17)$ & $102 \pm 11(6)$ & $124 \pm 37(11)$ & 0.07 & $122 \pm 36(12)$ & $101 \pm 12(5)$ & 0.14 \\
\hline $\mathrm{C} 4, \mathrm{mg} / \mathrm{dL}$ & $30 \pm 9(17)$ & $33 \pm 9(6)$ & $29 \pm 10(11)$ & 0.72 & $28 \pm 10(12)$ & $35 \pm 8(5)$ & 0.32 \\
\hline \multicolumn{8}{|l|}{ Hypocomplementemia, } \\
\hline Malignancy & $15(40)$ & $10(10)$ & $17(29)$ & 1 & 16 & 14 & 1 \\
\hline COPD & $13(40)$ & $30(10)$ & $7(29)$ & 0.10 & 11 & 43 & 0.10 \\
\hline CAD & $20(40)$ & $10(10)$ & $24(29)$ & 0.65 & 26 & 14 & 0.65 \\
\hline AI & $13(40)$ & $20(10)$ & $10(29)$ & 0.59 & 21 & 0 & 0.55 \\
\hline $\mathrm{DM}$ & $28(40)$ & $30(10)$ & $24(29)$ & 0.69 & 16 & 43 & 0.30 \\
\hline \multicolumn{8}{|l|}{ Outcomes, $\%$} \\
\hline Dialysis & 36 & 82 & 30 & 0.004 & 26 & 89 & 0.02 \\
\hline Transplant & 10 & 0 & 13 & 0.56 & 6 & 0 & 1 \\
\hline Death & 24 & 36 & 20 & 0.41 & 21 & 43 & 0.34 \\
\hline
\end{tabular}

Mean $\pm \mathrm{SD},(n)$ given if less than $n$ in header row, bolded $p$ value denotes significance.

† Fisher's exact test for categorical variables (\%), Mann-Whitney test for continuous variables.

eGFR calculated using MDRD equation, other or unreported race $n=1$ data not shown.

GFR, glomerular filtration rate; BUN, blood urea nitrogen; HDL, high-density lipoprotein; LDL, low-density lipoprotein; TG, triglycerides; BP, blood pressure; WBC, white blood cell; HCT, hematocrit; ANA, anti-nuclear antibody; C in C3/4, complement; SPEP, serum protein electrophoresis; UPEP, urine protein electrophoresis; SLC, serum light chain; HIV, human immunodeficiency virus; $\mathrm{COPD}$, chronic obstructive pulmonary disease; $\mathrm{CAD}$, coronary artery disease; $\mathrm{AI}$, autoimmune disease; $\mathrm{DM}$, diabetes mellitus. 
Table 3. Comorbidities, treatments and outcomes of patients with fibrillary glomerulonephritis at UNC Kidney Center compared to other cohorts

\begin{tabular}{|c|c|c|c|c|c|}
\hline & $\begin{array}{l}\text { Rosenstock } \\
\text { et al. [1], } 2003 \\
(n=61)\end{array}$ & $\begin{array}{l}\text { Nasr et al. } \\
{[22], 2011} \\
(n=61)\end{array}$ & $\begin{array}{l}\text { Kalbermatter } \\
\text { et al. [8], } 2012 \\
(n=16)\end{array}$ & $\begin{array}{l}\text { Javaugue } \\
\text { et al. [23], } 2013 \\
(n=27)\end{array}$ & $\begin{array}{l}\text { UNC Kidney Center } \\
2017(n=42)\end{array}$ \\
\hline Autoimmune disease & $5(3)$ & $15(10)$ & $13(2)$ & $30(8)$ & $13(5 / 40)$ \\
\hline Hepatitis B & - & - & - & - & $8(2 / 26)$ \\
\hline Hepatitis C & $17(10)$ & $3(2)$ & - & $7(2)$ & $27(7 / 26)$ \\
\hline COPD & - & $5(3)$ & - & $19(5)$ & $13(5 / 40)$ \\
\hline Coronary artery disease & - & $9(6)$ & - & - & $18(7 / 39)$ \\
\hline Diabetes mellitus & $20(12)$ & $20(13)$ & $6(1)$ & $22(6)$ & $28(11 / 40)$ \\
\hline \multicolumn{6}{|l|}{ Treatment, $\%(n)$} \\
\hline No treatment & - & $26(16)$ & - & - & $7(3)$ \\
\hline Immunosuppressive therapy, \% ( $n)$ & $n=20(33 \%)$ & $n=29(44 \%)$ & $n=13(81 \%)$ & $n=13(48 \%)$ & $n=20(48 \%)$ \\
\hline Rituximab & - & $10(3)$ & - & $54(7)$ & $75(15)$ \\
\hline Steroids included & - & $83(24)$ & - & - & $40(8)$ \\
\hline Steroids alone & $45(9)$ & $28(8)$ & $62(8)$ & $38(5)$ & $5(1)$ \\
\hline Cyclosporine & $15(3)$ & $7(2)$ & $8(1)$ & $31(4)$ & $20(4)$ \\
\hline Cyclophosphamide & $40(8)$ & $31(9)$ & $38(5)$ & $23(3)$ & $10(2)$ \\
\hline MMF & - & $21(6)$ & - & $8(1)$ & $5(1)$ \\
\hline Bortezomib & - & - & - & $8(1)$ & $10(2)$ \\
\hline Outcome, \% (n) & $n=60$ & $n=61$ & $n=16$ & $n=27$ & $n=42$ \\
\hline Dialysis/ESKD & $45(28)$ & $44(27)$ & $38(6)$ & $48(13)$ & $36(15)$ \\
\hline Transplant & $4(2)$ & $23(14)$ & - & - & $10(4)$ \\
\hline Death & - & $20(12)$ & $38(6)$ & $19(5)$ & $24(10)$ \\
\hline
\end{tabular}

COPD, chronic obstructive pulmonary disease; MMF, mycophenolate mofetil.

for IgG (kappa and lambda) in 95\% of biopsies, followed closely by C3 (positive in $91 \%$ of biopsies), C1q (65\%), $\operatorname{IgM}(56 \%)$ and $\operatorname{IgA}(39 \%)$.

\section{Treatments}

In our cohort, 3 patients (7\%) received no treatment due to the severity of kidney disease and significant degree of tubulointerstitial scarring at the time of presentation deeming the treatment futile. Hemodialysis was initiated in 3 patients (7\%) at the time of presentation. Renin-angiotensin-aldosterone system (RAAS) blockade alone was instituted in $24 \%$ of patients $(n=10)$, and RAAS blockade in conjunction with an immunomodulating agent was prescribed in $36 \%(n=15)$ of patients.

Five prior studies reported treatment data on the use of immunosuppressive therapy (Table 3). We found that the inclusion of corticosteroids in the treatment plan was less common in our cohort compared to others. There has been a notable increase in rituximab use over time, with $17 \%$ of patients receiving rituximab in 2011, 54\% in 2013 and $75 \%$ in our group $[22,23]$.

In our cohort, 15 patients were advised to initiate rituximab (online suppl. Table 3). Nine patients were classified by outcome as a result of treatment. Outcomes were similar to previously reported rituximab data as $11 \%$ were non-progressive, $56 \%$ were progressive and $33 \%$ reached ESKD [21]. Patients in our cohort whose outcome was ESKD had a longer time from biopsy to rituximab dose ( 30 vs. 4.5 months, $p=0.05$ ), higher average creatinine at the time of rituximab treatment (3.22 vs. $2.27 \mathrm{mg} / \mathrm{dL}, p=0.38$ ), and the initial biopsy revealed a higher percentage of sclerosis ( 49 vs. $22 \%, p=$ $0.02)$. 


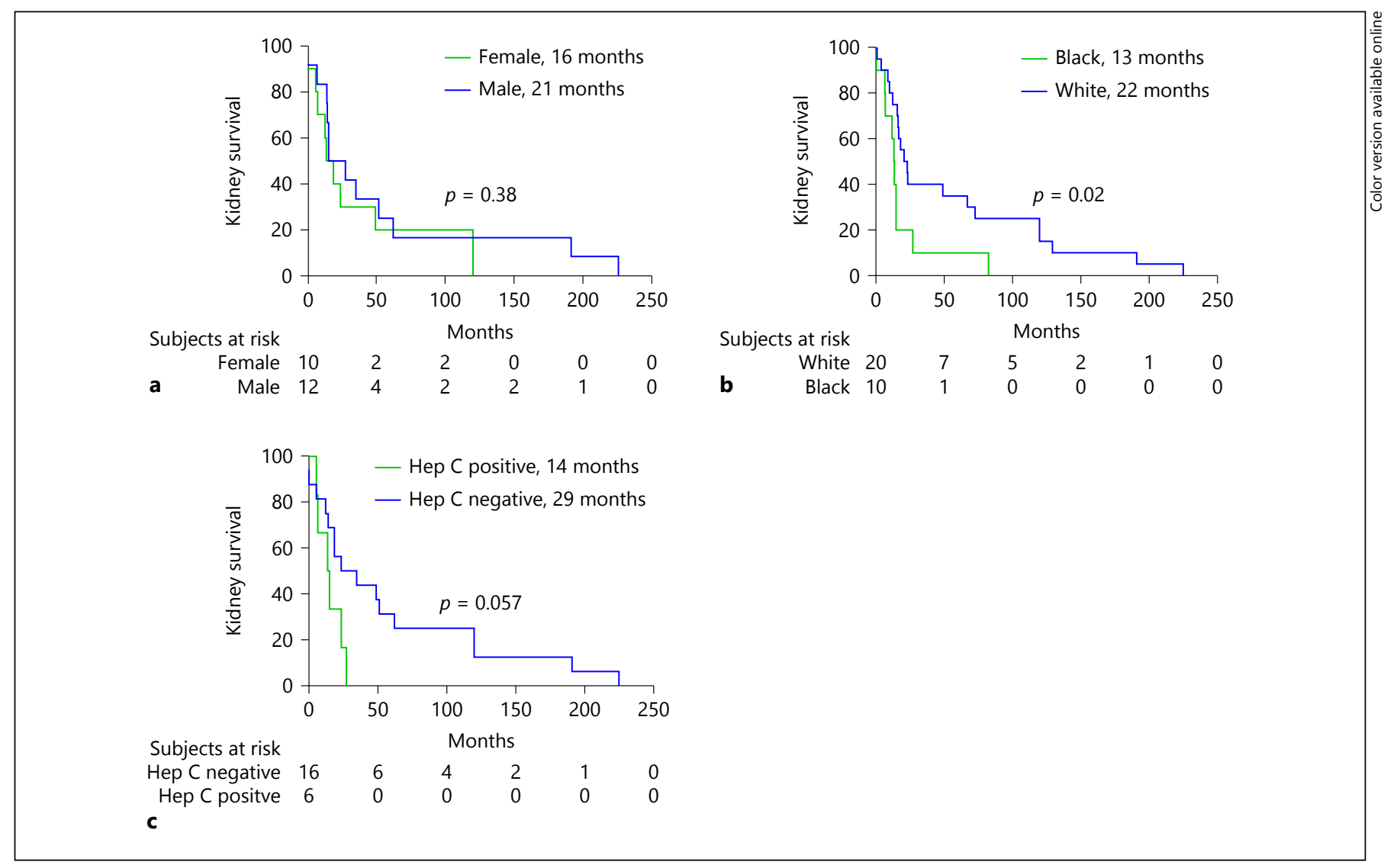

Fig. 1. a-c Kaplan-Meier estimates of significant loss of kidney function. Kidney function was defined as impaired if there was a doubling of serum creatinine since biopsy, initiation of dialysis, transplant or death. Estimated glomerular filtration rate at diagnosis was similar between groups.

\section{Outcomes}

Despite having a higher average serum creatinine at the time of diagnosis, patients in our cohort had similar patient and kidney survival when compared to prior reports (Table 3). Of patients that are still being managed by our group, $6(19 \%)$ are on dialysis with a 13-month median time to dialysis initiation (15/42 patients) for our entire cohort. Twenty-three percent $(n=7)$ of patients were lost to follow-up or have not been seen in the UNC Hospitals system within the last 2 years. Two patients have had a transplant without recurrence and $52 \%$ of patients in the cohort are still actively followed and are free from ESKD or doubling of creatinine. Of these 16 patients, the median time from diagnosis to last visit was 16 months (interquartile range 9-82) and the mean creatinine was $2.5 \pm 1 \mathrm{mg} / \mathrm{dL}$, glomerular filtration rate (GFR) was $29 \pm 15$ and UPC was $4.6 \pm 7$.

The rate of primary renal outcome (a composite of doubling of serum creatinine, initiation of dialysis, trans- plant or death) was similar in males and females ( $p=0.38$; Fig. 1a), but was significantly higher in black compared to white patients ( $p=0.02$; Fig. 1b). Kidney survival was not significantly different between $\mathrm{HCV}$-positive and HCV-negative patients, but the small sample size limited this evaluation ( $p=0.06$; Fig. 1c). There was no difference in kidney survival in HCV-negative black versus white patients $(p=0.22)$.

\section{Discussion}

In this study, we report the clinical features and outcomes of patients with fibrillary glomerulonephritis diagnosed in the southeastern United States and compare patient characteristics in this cohort with those of patients described in prior reports. Prior studies have predominantly focused on patients of European descent $[1,8,21-$ $24]$, and this is the first study to focus on patients in the 
southeastern United States, where the black race is prevalent. Compared to prior cohorts, our patients were more likely to be black and have coexisting HCV infection. Black patients were more likely to be transitioned to dialysis and have decreased kidney survival time compared to white patients. Patients with HCV were more likely to progress to ESKD and transition to dialysis compared to patients without HCV. However, black patients without HCV had similar outcomes compared to white patients without HCV. Although black patients represent $12 \%$ of the United States population, they comprise $22 \%$ of HCV-positive individuals [25]. The percentage of black patients with fibrillary glomerulonephritis treated in our clinic closely reflects the racial composition of the southeastern United States [26]. Unlike other glomerular diseases such as lupus nephritis, membranous nephropathy and ANCA glomerulonephritis that have a predilection for specific racial groups, our cohort demonstrates that fibrillary glomerulonephritis is evenly distributed among patients of different races, a finding not reported earlier.

Our study also supports an association between fibrillary glomerulonephritis and infections, malignancy and autoimmune disorders, as over half of our patients had one of these comorbidities, higher than previously reported. Furthermore, a recent study of 66 patients with fibrillary glomerulonephritis from the Mayo Clinic (95\% Caucasians) found that one third of patients had malignancy, autoimmune disease or monoclonal gammopathy [22]. It was proposed that while fibrillary glomerulonephritis has been traditionally considered a primary glomerular disease, all patients should be screened for secondary causes as part of their initial evaluation. This proposed recommendation has both diagnostic and therapeutic implications, as treatment of the associated comorbidity may lead to the resolution of the glomerular disease process.

An association between HCV infection and fibrillary glomerulonephritis has been previously described [27, 28]. However, our cohort is unique in that one third of patients tested were positive for $\mathrm{HCV}$, which is much higher than previously reported. While the exact etiology of fibrillary glomerulonephritis is largely unknown, pathogenesis likely relates to the deposition of immune complexes containing IgG and complement: $\mathrm{HCV}$ is a plausible candidate to elicit such an immune response [27]. All of our patients with HCV were diagnosed before the availability of direct-acting antiviral therapy, and none were treated with older agents for a sustained period of time. Neither were any of these patients treated with rituximab, which was used in the treatment of HCV-re- lated cryoglobulinemic glomerulonephritis. Future studies are warranted to investigate the presence of $\mathrm{HCV}$ viral antigens within the immune deposits in fibrillary glomerulonephritis, and to explore the use of antiviral therapies in eradicating glomerular disease activity.

Since fibrillary glomerulonephritis involves glomerular deposition of immunoglobulin, a rationale for the use of the CD20-positive B-cell depleting agent rituximab exists, especially in patients with comorbid humoral autoimmune disease. Published outcomes of rituximab treatment are varied. In a study of 3 adults (presenting with serum creatinine from 0.6 to $1.3 \mathrm{mg} / \mathrm{dL}$ with a follow-up of 9-46 months), kidney disease was stabilized [29]. The outcomes of a study of 7 patients (average estimated GFR [eGFR] of $66 \mathrm{~mL} / \mathrm{min} / 1.73 \mathrm{~m}^{2}$, mean follow-up of 44 months) were 2 patients with no response, one with complete response and 4 with partial response to rituximab [23]. Twelve patients treated with rituximab (average serum creatinine of $2.1 \mathrm{mg} / \mathrm{dL}$ and eGFR of $39 \mathrm{~mL} /$ $\min / 1.73 \mathrm{~m}^{2}$ ) resulted in $33 \%$ of patients with stabilized kidney function, however, these patients had lower serum creatinine levels prior to treatment (average $1.25 \mathrm{mg} / \mathrm{dL}$ ). Patients whose kidney function deteriorated $(25 \%)$ had a mean serum creatinine of $1.93 \mathrm{mg} / \mathrm{dL}$, and patients that progressed to ESKD (42\%) had a mean serum creatinine of $2.5 \mathrm{mg} / \mathrm{dL}$ at presentation. In our cohort, delayed initiation of rituximab treatment, increased creatinine at the time of treatment and higher rates of sclerosis at the time of biopsy were more likely to result in a patient with an outcome of ESKD (online suppl. Table 3). Given that many of our patients received other treatments in addition to rituximab, we speculate that treatment with rituximab slows the progression to ESKD and may be beneficial if used swiftly after disease diagnosis.

Our retrospective study has several limitations. Complete demographic and clinical characteristics of all 287 patients whose native kidney biopsies were interpreted at UNC are lacking; however, age, race, sex, entry serum creatinine, eGFR, serum albumin and proteinuria were not statistically different between this larger group and the 42 patients who were treated at UNC and further analyzed. Our findings also reflect the treatment practices of one institution, and cannot necessarily be generalized to other patient cohorts. Few patients received rituximab, and those who did were mainly treated in the more modern era, limiting their follow-up duration. None of the HCV patients were treated with newer antiviral agents; thus, we cannot determine whether effective eradication of the virus might lead to glomerular disease resolution. Finally, our sample size was small - due to the rarity of this dis- 
ease - and thus our statistical power to detect betweengroup differencesby race, HCV status and sex, was limited.

Our findings provide new insights into the demographic and clinical characteristics of patients with fibrillary glomerulonephritis, and explore the influence of race and comorbidity on disease onset and progression. A distinct subgroup of patients of black race with co-existing HCV infection, who appear to have a particularly poor prognosis, was identified for the first time. This vulnerable patient group may benefit from a combination of antiviral and rituximab therapies to eradicate their immunecomplex mediated glomerular disease, although this hypothesis requires further study. Importantly, our findings also suggest that screening for infections, malignancy and autoimmune diseases may be warranted in all patients presenting with this rare disease entity, as the potential for fibrillary glomerulonephritis to be a secondary disease process may have been under-appreciated in prior reports. In addition, proteomic profile studies of the deposits in fibrillary glomerulonephritis suggest that the presence of apolipoprotein $\mathrm{E}$ and immunoglobulin light or heavy chain $\mathrm{C}$ region may be key to the organization and tissue deposition, making these potential targets of future therapies [30]. Further investigation into disease patho- genesis and appropriate treatment through multi-center collaborations and well-conducted randomized controlled clinical trials is warranted.

\section{Acknowledgements}

The authors wish to thank the patients with fibrillary glomerulonephritis and all those dedicated to their care at UNC and Daniel Kenan of the UNC Department of Pathology for histopathology images, Michelle O'Shaughnessy of Stanford Department of Nephrology for critical and constructive review of the manuscript, Yichun Hu of the UNC Kidney Center for initial statistical assistance and the GDCN for providing the long-standing infrastructure to make this work possible. A portion of the results were presented in abstract form at ASN Kidney Week 2015. F.P.S. is supported by NIDDK grant T32 \#5T32DK007750-16. W.F.P., C.J.P., J.C.J., R.J.F., and P.H.N. are supported by grants from the NIDDK (P01-DK058335-15 and 1UM1-DK100845-01). W.F.P. was also supported in part by NIDDK grant \#5F32DK097891-02 and received translational research funding from the Broad Institute (Cambridge, MA, USA).

\section{Disclosure Statement}

The authors report no conflict of interest.

\section{References}

1 Rosenstock JL, Markowitz GS, Valeri AM, Sacchi G, Appel GB, D’agati VD: Fibrillary and immunotactoid glomerulonephritis: distinct entities with different clinical and pathologic features. Kidney Int 2003;63:1450-1461.

2 Fogo A, Qureshi N, Horn RG: Morphologic and clinical features of fibrillary glomerulonephritis versus immunotactoid glomerulopathy. Am J Kidney Dis 1993;22:367-377.

3 Rosenmann E, Eliakim M: Nephrotic syndrome associated with amyloid-like glomerular deposits. Nephron 1977;18:301-308.

4 Iskandar SS, Falk RJ, Jennette JC: Clinical and pathologic features of fibrillary glomerulonephritis. Kidney Int 1992;42:1401-1407.

5 Duffy JL, Khurana E, Susin M, Gomez-Leon G, Churg J: Fibrillary renal deposits and nephritis. Am J Pathol 1983;113:279-290.

6 Lusco M, Fogo AB, Najafian B, Alpers CE: $\mathrm{AJKD}$ atlas of renal pathology: fibrillary glomerulonephritis. Am J Kidney Dis 2015; 66:e27-e28

7 Alpers CE, Rennke HG, Hopper J Jr, Biava CG: Fibrillary glomerulonephritis: an entity with unusual immunofluorescence features. Kidney Int 1987;31:781-789.

8 Kalbermatter SA, Marone C, Casartelli D, Hausberg M, Banfi G, Mihatsch M, et al: Outcome of fibrillary glomerulonephritis. Swiss Med Wkly 2012;142:w13578.
9 Nakhoul GN, Simon JF: Fibrillary glomerulonephritis associated with limited scleroderma: a case report. Clin Nephrol 2016;85:235237.

10 Amir-Ansari B, O’Donnell P, Nelson SR, Cairns HS: Fibrillary glomerulonephritis in a patient with adenocarcinoma of stomach. Nephrol Dial Transplant 1997;12:210211.

11 Miadonna A, Salmaso C, Palazzi P, Elli A, Braidotti P, Lambertenghi Deliliers G: Fibrillary glomerulonephritis in Castleman's disease. Leuk Lymphoma 1998;28:429-435.

12 Whitaker G, Brown EA, Moss J, Woodrow D, Frankel A: Fibrillary glomerulonephritis occurring in association with hereditary angioneurotic oedema, pernicious anaemia and hypothyroidism. Nephrol Dial Transplant 1998; 13:1822-1824.

13 Haas M, Rajaraman S, Ahuja T, Kittaka M, Cavallo T: Fibrillary/immunotactoid glomerulonephritis in HIV-positive patients: a report of three cases. Nephrol Dial Transplant 2000;15:1679-1683.

14 Yun YS, Song HC, Lee K, Choi EJ, Kim YS, Min JK, et al: Fibrillary glomerulonephritis in rheumatoid arthritis. Nephrology (Carlton) 2010;15:266-267.

15 Abraham G, Bargman JM, Blake PG, Katz A, Oreopoulos DG: Fibrillary glomerulonephri- tis in a patient with metastatic carcinoma of the liver. Am J Nephrol 1990;10:251-253.

16 Alpers CE, Kowalewska J: Fibrillary glomerulonephritis and immunotactoid glomerulopathy. J Am Soc Nephrol 2008;19:34-37.

17 Bridoux F, Hugue V, Coldefy O, Goujon JM, Bauwens M, Sechet A, et al: Fibrillary glomerulonephritis and immunotactoid (microtubular) glomerulopathy are associated with distinct immunologic features. Kidney Int 2002;62:1764-1775.

18 Ivanyi B, Degrell P: Fibrillary glomerulonephritis and immunotactoid glomerulopathy. Nephrol Dial Transplant 2004;19:2166-2170.

19 Korbet SM, Schwartz MM, Lewis EJ: The fibrillary glomerulopathies. Am J Kidney Dis 1994;23:751-765.

20 Iskandar SS, Falk RJ, Jennette JC: Clinical and pathologic features of fibrillary glomerulonephritis. Kidney Int 1992;42:1401-1407.

21 Hogan J, Restivo M, Canetta PA, Herlitz LC, Radhakrishnan J, Appel GB, et al: Rituximab treatment for fibrillary glomerulonephritis. Nephrol Dial Transplant 2014;29:19251931.

22 Nasr SH, Valeri AM, Cornell LD, Fidler ME, Sethi S, Leung N, et al: Fibrillary glomerulonephritis: a report of 66 cases from a single institution. Clin J Am Soc Nephrol 2011;6: 775-784. 
23 Javaugue V, Karras A, Glowacki F, McGregor B, Lacombe C, Goujon JM, et al: Long-term kidney disease outcomes in fibrillary glomerulonephritis: a case series of 27 patients. Am J Kidney Dis 2013;62:679-690.

24 Mallett A, Tang W, Hart G, McDonald SP, Hawley CM, Badve SV, et al: End-stage kidney disease due to fibrillary glomerulonephritis and immunotactoid glomerulopathy outcomes in 66 consecutive ANZDATA registry cases. Am J Nephrol 2015;42:177-184.
25 Pearlman BL: Hepatitis $C$ virus infection in African Americans. Clin Infect Dis 2006;42: 82-91.

26 US Census Bureau: The Black Population: 2010, 2012, pp 1-20.

27 Markowitz GS, Cheng JT, Colvin RB, Trebbin WM, D'Agati VD: Hepatitis C viral infection is associated with fibrillary glomerulonephritis and immunotactoid glomerulopathy. J Am Soc Nephrol 1998;9:2244-2252.

28 Coroneos E, Truong L, Olivero J: Fibrillary glomerulonephritis associated with hepatitis C viral infection. Am J Kidney Dis 1997;29: 132-135.
29 Collins $M$, Navaneethan SD, Chung M, Sloand J, Goldman B, Appel G, et al: Rituximab treatment of fibrillary glomerulonephritis. Am J Kidney Dis 2008;52:11581162.

30 Sethi S, Theis JD, Vrana JA, Fervenza FC, Sethi A, Qian Q, et al: Laser microdissection and proteomic analysis of amyloidosis, cryoglobulinemic GN, fibrillary GN, and immunotactoid glomerulopathy. Clin J Am Soc Nephrol 2013;8:915-921. 